\title{
DIVERSIDADE DE PARASITÓIDES DE PHYLLOCNISTIS CITRELLA LEPIDOPTERA: GRACILLARIIDAE) EM VARIEDADES DE CITROSE A RELAÇÃO COM FATORES BIÓTICOSE ABIÓTICOS
}

\section{C.F.S. Efrom ${ }^{1}$, L.R. Redaelli', L.M .G. D iefenbach ${ }^{2}$}

IUniversidadeFederal do Rio Grandedo Sul, FaculdadedeA gronomia, Departamento deFitotecnia, A v. Bento Gonçalves, 7712, CEP 91540-000, Porto A legre, RS, Brasil. E-mail: caioefrom@hotmail.com

\section{RESUMO}

A diversidadedeparasitóides do minador-dos-citros, Phyllocnistiscitrella (Lep.:Gracillariidae), bem como a relação destes insetos com fatores bióticos e abióticos, foram avaliados por amostragensquinzenais, dejulho/ 2003ajunho/ 2005, em 2 pomares sob manejo orgânico, um de tangerineira 'M ontenegrina' (Citrus deliciosa) e outro do tangoreiro 'Murcott' (C. sinensis $\times C$. reticulata), em M ontenegro ( $29^{\circ} 37^{\prime} 51^{\prime \prime} \mathrm{S}, 51^{\circ} 28^{\prime} 10^{\prime \prime} \mathrm{W}$ ), RS. Em cada ocasião deamostragem, em 24 plantas sorteadas, examinaram-se todas as folhas contendo câmaras pupais do minador. Em laboratório, a porção da fol ha com a câmara foi individual izada emtubos deensaio, mantidos em câmara climatizada atéa emergência dos parasitóides ou deP. citrella. Estimou-se, nos dois anos, o número de brotos das copas das plantas. A geniaspis citricola foi o parasitóide mais abundante, representando mais de $80 \%$ do total de parasitóides nas duas variedades e diferentes anos. Os outros parasitóides registrados foram Cirrospilus neotropicus, Cirrospilus floridensis, Elasmus phyllocnistoides e Chrysocharis vonones, este último constitui o primeiro registro desta espécie atuando sobre P. citrella. A diversidade de parasitóides foi semel hante entre as variedades e diferente entre os anos. A temperatura máxima teve maior influência sobre o minador e os parasitóides, mas após o estabelecimento, P. citrella também apresenta correlação com os fluxos de brotação. Os períodos de estiagens durante o trabal ho podem ter influenciado as populações do minador e de parasitóides por diminuírem os recursos disponíveis para ambos os grupos.

PALAVRAS-CHAVE: Parasitismo, minador-dos-citros, fatores climáticos.

\section{ABSTRACT}

DIVERSITY OFPHYLLOCNISTISCITRELLA (LEPIDOPTERA:GRACILLARIIDAE) PARASITOIDS IN CITRUS CULTIVARSAND THE RELATIONSHIP WITH BIOTIC AND ABIOTIC FACTORS. The diversity of parasitoids of thecitrusleafminer, Phyllocnistis citrella (Lep.: Gracillariidae), aswell astheir relationship with bioticand abioticfactors, wereevaluated through fortnightly samplings, fromJuly/ 2003 to June/ 2005, in two organically managed orchards, one of "Montenegrina" tangerine (Citrus deliciosa) and the other of the "Murcott" tangor (C. sinensis XC. reticulata), in Montenegro (29 $37^{\prime} 51^{\prime \prime} \mathrm{S}$, $51^{\circ} 28^{\prime} 10^{\prime \prime}$ W), state of Rio Grande do Sul, Brazil. A teach sampling occasion, in 24 randomly selected trees, all leaves containing pupal chambers of theleaf miner wereexamined. Inthelaboratory, theleaf portion with the chamber was confined in a test tubeand kept under controlled conditions until the emergence of the parasitoids or P. citrella. Thenumber of shoots in thetreecanopy was estimated for both sampling years. A geniaspis citricola wasthemostabundantparasitoid, representing morethan $80 \%$ of thetotal parasitoidsinthetwocultivarsinthesetwoyears. Other registered parasitoid specieswere: Cirrospilus neotropicus, Cirrospilus floridensis, Elasmus phyllocnistoides and Chrysocharis vonones, thelatter constituting the first register of this species on P. citrella. Parasitoid diversity was similar among cultivars and different among years. The maximum temperature had the greatest influence on the miner and its parasitoids. N evertheless, after its establishment, $P$. citrella al so shows correlation with the young flush. The drought periods occurring during the study might have influenced the citrus leafminer and parasitoids populations due to the reduction of available resources for both groups.

KEY WORDS: Parasitism, citrus leafminer, meteorological factors.

2Fundação Estadual deProdução ePesquisa em Saúde, Instituto de Pesquisas Biológicas, Laboratório Central de Saúde Pública, Porto A legre, RS, Brasil. 


\section{INTRODUÇÃO}

Phyllocnistis citrella Stainton, conhecida popularmentecomo minador-dos-citros, éconsideradapraga eocorreem praticamentetodos os países produtores decitros (HEPPNER, 1993). A fêmeaovipositanasfol has novas dos brotos e as lagartas penetram no tecido foliar, alimentando-se do parênquima lacunoso, re sultando numa injúria caracterizada por galerias sinuosas cobertas pela epiderme, com aspecto semeIhanteaumapelículaprateada(HEPPNER, 1993; GARIJO; García, 1994; Generalitat Valenciana, 1996). A injúria cria um microambiente favorável ao desenvolvimento de patógenos como a bactéria causadora do cancro cítrico ( $X$ anthomonas citri pv. ditri), uma das principais doenças dos citros (Graham et al., 1996; Chagas et al., 2001; Amaral, 2003).

Tendo em vistao hábito minador deP .citrella, oseu controle tem sido bastante difícil. Assim, inúmeras alternativas vêmsendo buscadas, incluindoaimportação do parasitóide exótico Ageniaspis citricola Logvinovskaya(Hymenoptera: Encyrtidae), suacriação massal e liberação (Neale et al., 1995; Argov; Rössler, 1996; Pomerinke \& Stansly, 1998; Paiva et al., 2000; URBANEjA et al., 2000). Poucostrabal hostiveram como objetivo avaliar a diversidade, a freqüência ea contribuição de inimi gos naturais nativos já presentes nos agroecossistemas e que poderiam atuar ou já estariam atuando sobre P. citrella. De acordo com URBANEjA et al. (1998) a adaptação de parasitóides autóctones oportunistas, bem como a utilização de inimigos naturais específicos, podeser fundamental na regulação das populações desta praga.

Entre os inimigos naturais registrados atuando sobre P. citrella destacam-se os himenópteros parasitóides das famílias Eulophidae, Chalcididae, Eupel midaee Encyrtidae (Schaufr et al., 1998; Lega SPI et al., 1999; N Ascimentoetal., 2000; JAHNKE et al.,2005b).

Considerando-sequea contribuição destesinimigos naturais depende, dentre outros aspectos, das variações espaciais etemporais de suas populações, éimportanteconhecer ainfluênciaquefatoresbióticos e abióticos exercem nestas variações. O presente trabal ho apresenta os resultados da diversidade e freqüência deespécies de parasitóides nativos eexóticos associados aP . citrella, empomaresorgânicosde citros do Vale do Caí, RS, bem como as relações entre a preci pitação pluviométrica, a temperatura do ar, a brotação e as populações do hospedeiro e de seus parasitóides.

\section{MATERIAL E MÉTODOS}

O experimento foi realizado em Montenegro, RS, ondeo dima, segundo a dassificação deKöppen, édo tipo Cfa, subtropical úmido com precipitações bem distribuídas, sofrendo a influência de massas de ar tropicaisepolar-atlânticas( M ALUF, 2000). A temperaturamédiaanual éde $19,1^{\circ} \mathrm{C}$, comamáximamédiade $25,8^{\circ}$ C e mínima média de $14,4^{\circ} \mathrm{C}$. A precipitação pluviométricamédiaéde $1.424 \mathrm{~mm} /$ ano (IPAGRo, 1989).

O trabal ho foi desenvolvido em dois pomares de

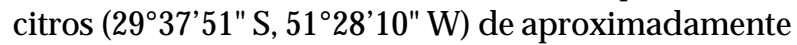
0,6 ha cada, um da variedade de tangoreiro Murcott (Citrus sinensis L. Osbeck $x$ Citrus reticulata Blanco) eo outro de tangerineira Montenegrina Citrus deliciosa Tenore). Os pomares com 312 plantas cada, de 14 anos de idade, são mantidos sob manejo orgânico desde a instalação, com aplicações de chorume e composto orgânico provenientes da Usina de Compostagem da CooperativadosCitricultoresEcológi cosdoValedoCaí (Ecocitrus), além dautilização decaldabordalesa(0,5\%) três vezes por ano (setembro, novembro edezembro).

As amostragensforam real izadasquinzenalmente, de julho de 2003 a junho de 2005, quando se examinavam todas as folhas da planta contendo câmaras pupais do minador, de 24 plantas previamente sorteadas. As folhas foram acondicionadas individualmenteem sacos depolietileno etiquetados e transportadas em caixa de poliestireno contendo "termogel", para que não perdessem a turgidez.

Em laboratório, as fol has foram examinadas com auxílio demicroscópioestereoscópioeaporção dafolha quecontinha a câmara pupal era recortada eindividualizada em tubo de ensaio $(10 \mathrm{~mm} \varnothing \times 40 \mathrm{~mm}$ de comprimento) contendo, no fundo, al godão umedecido comáguadestilada. Ostuboserammantidosemcâmara climatizada $\left(25 \pm 2^{\circ} \mathrm{C}\right.$; fotofase $\left.12 \mathrm{~h}\right)$ atéaemergênciados parasitóidesou deP.citrella. Diariamenteosparasitóides emergidos foram mortos e mantidos individualizados emálcool 70\% para posterior identificação. Os adultos deP . citrella forammortoseacondicionadosemenvelope entomológico identificado.

A identificação dos parasitóides, a nível de família, foi realizada com auxílio de chave dicotômica (Schauff et al., 1998) ea específica, por especialista.

Nas ocasiões deamostragem, realizou-sea contagemdo número debrotos dacopa da planta, utilizando-seuma moldura $(25 \times 25 \mathrm{~cm})$. Para uniformizar a contagem, dividiu-seacopadaplantaem4quadrantes (Norte, Sul, Lestee Oeste) edois estratos, inferior (0-1 m) esuperior (1,01-2m), consideradosapartir dabase da copa. O número de brotos presentes na área limitada pela moldura era contado, nas 8 diferentes parcel as da copa de seis plantas, de cada uma das variedades. N estas mesmas plantas, media-se a al tura e a circunferência da copa utilizando-se fita métrica e, estimava-seassim, onúmero total debrotos paratoda a copa da planta.

A diversidade de parasitóides foi avaliada pelos índices de Shannon-Wiener $\left(\mathrm{H}^{\prime}\right)$ e o de diversidade 
(ou complementar) de Simpson (1-D) (KreBS, 1998), comparadas pelo teste de Bootstrapping, conforme MoRENo (2001). Utilizou-se para estes cálculos o programa Past versão 1.46 (H Ammer et al., 2001).

Para comparação dos fluxos de brotação entre anosevariedadesutilizou-seotestedec ${ }^{2}$. A influência dos fatores bióticos eabióticos sobre os parasitóides foi avaliadaatravés decorrelação dePearsoneregressãolinear simplesemúltiplapelo método "stepwise" eonível designificânciaadotadofoi de5\%(CALLEGARIJACQUES, 2003). Utilizou-separa ostestes estatísticoso programa N CSS-PASS 2004 (H INTZE, 2004).

Para as correlações e regressões, utilizaram-se os dados diáriosmédios detemperatura média, mínima e máxima $\left({ }^{\circ} \mathrm{C}\right)$, além da soma da precipitação pluviométrica $(\mathrm{mm})$ da quinzena anterior à amostragem, obtidos junto à Estação Meteorológica da Fundação Estadual de Pesquisa Agropecuária (FEPA GRO), em Taquari, RS, Município situado na mesmaregião ecofisiográfica, aaproximadamente30 km do experimento.

\section{RESULTADOSE DISCUSSÃO}

Foram real izadas 52 amostragens ao longo dos dois anos e através do número médio de brotos identificaram-se três fluxos de brotação, tanto em 'Montenegrina', quanto em 'Murcott' (Fig. 1). Em 'Murcott', observou-se o mesmo, embora os valores totais tenham sido menores, corroborando o referido por Rodrigues; Dornelles (1999) que afirmam que 'Montenegrina' possui copa densamente foliada.

As plantas de 'M urcott' têm arquitetura diferente das de 'M ontenegrina', a copa é mais alta e aberta, al ém de possuírem fol has maiores. Entretanto, não foram encontradas diferenças significativas entre o númeromédio debrotos por plantadasduasvariedades e nos dois anos $\left(\chi^{2}=0,939 \mathrm{P}=0,3327\right)$.

No período de julho de 2003 a junho de 2004 (primeiroano), amostraram-se, nos doispomares, 289 câmaras pupais. Daquelas oriundas de 'Montenegrina', emergiram 65 adultos deP. citrella e 55 parasitóides, das provenientes de 'M urcott', obteve-se 33 adultos de P. citrella e 22 parasitóides. No segundo ano, de julho de 2004 a junho de 2005, coletaram-se 581 câmaras pupais e registrou-se a emergência, em 'Montenegrina', de 104 adultos deP . citrella e 257 parasitóides e, em 'M urcott', 48 adultos deP. citrella e 136 parasitóides.

Os parasitóides encontrados pertencem àsfamílias de Chalcidoidea: Encyrtidae (A geniaspis citricola) e Eulophidae Cirrospilus neotropicus Diez \& Fidalgo, Cirrospilus floridensis Evans, Chrysocharis vononesWalker eElasmus phyllocnistoides Diez, Torréns \& Fidalgo).

A . citricol a foi aespéciemaisabundante, representando mais de $80 \%$ dos parasitóides, nos dois anose nas duas variedades (Tabela 1), provavel mentedevido a sua alta especificidade com o hospedeiro, por apresentar poliembrionia, além dealtacapacidadede dispersão ede busca (H oY; N GUYEN, 1997; POMERINKE; STANSLY, 1998). J AHNKE etal. (2006), emtrabal ho desenvolvido namesmaregião do presenteestudo, registraram a mesma freqüência paraA . citricola.

$N$ as diversas regiões do mundo onde $P$. citrella ocorre, as espécies de parasitóides associadas emais comumente relatadas, pertencem a Eulophidae (LaSalle; Peña, 1997; Schauff et al., 1998; SÁ et al., 1999). Cirrospilus éum gênero numeroso, cosmopolita, com diversas espécies parasitando $P$. citrella (Schauff et al., 1998). SegundoDiez, Fidalgo (2003), são conhecidas três espécies de Cirrospilus como parasitóides de $P$. citrella nas Américas, duas das quais foram encontradas no presente estudo.

C. neotropicus apresentadistribuiçãoN eotropical ejá haviasidoregistradanoBrasil por SÁ et al. (1999) eJAHNKE et al. (2005b) (citada como Cirrospilus sp. C) e também para a Argentina, Colômbia, Honduras (Schaufr et al., 1998, comoCirrospilus sp.C)eM éxico( BAUTISTA-M ARTINEZ et al., 1996, citadaC .cuadristriatus). Tal como C.neotropicus, C. floridensis tem distribuição N eotropical, da Flórida ao sul do Brasil (JAHNKE et al., 2005a).

Tabela1- Número deindivíduos(N) efreqüênciarelativa(\%) (fr) das espécies deparasitói desencontradasnos pomares de Citrus deliciosa var. M ontenegrina e do tangoreiro 'M urcott' (Citrus sinensis x Citrus reticulata) em M ontenegro, RS, nos períodos de julho de 2003 a junho de 2004 (A no 1) e julho de 2004 a junho de 2005 (A no 2).

\begin{tabular}{|c|c|c|c|c|c|c|c|c|}
\hline \multirow{3}{*}{ Espécie } & \multicolumn{4}{|c|}{ 'Montenegrina' } & \multicolumn{4}{|c|}{ 'Murcott' } \\
\hline & \multicolumn{2}{|c|}{ Ano 1} & \multicolumn{2}{|c|}{ Ano 2} & \multicolumn{2}{|c|}{ Ano 1} & \multicolumn{2}{|c|}{ Ano 2} \\
\hline & $\mathrm{N}$ & $\operatorname{fr}(\%)$ & $\mathrm{N}$ & $\operatorname{fr}(\%)$ & $\mathrm{N}$ & $\operatorname{fr}(\%)$ & $\mathrm{N}$ & $\operatorname{fr}(\%)$ \\
\hline A geniaspis citricola & 55 & 93,22 & 210 & 81,71 & 22 & 100 & 120 & 88,23 \\
\hline Cirrospilus neotropicus & 4 & 6,78 & 35 & 13,62 & - & - & 7 & 5,15 \\
\hline Cirrospilus floridensis & - & - & 6 & 2,33 & - & - & 5 & 3,68 \\
\hline Chrysocharis vonones & - & - & 4 & 1,56 & - & - & 2 & 1,47 \\
\hline Elasmus phyllocnistoides & - & - & 2 & 0,78 & - & - & 2 & 1,47 \\
\hline
\end{tabular}



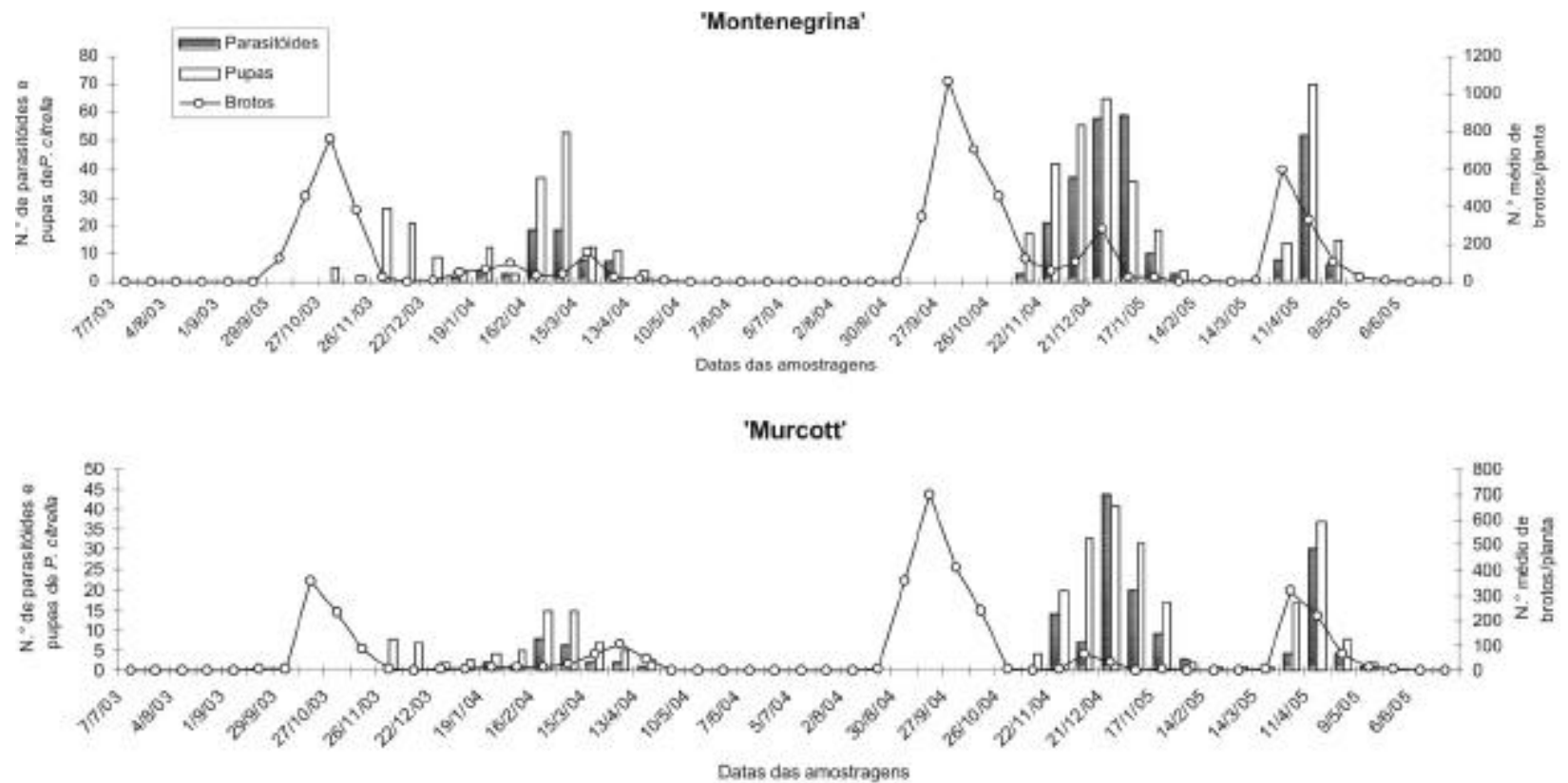

Fig. 1- Número depupas deP hyllocnistiscitrella, parasitóidesenúmeromédio debrotos/ planta registradosempomares de Citrus deliciosa var. Montenegrina e do tangoreiro 'M urcott' (Citrus sinensis X Citrus reticulata) em Montenegro, RS, no período dejulho de 2003 a junho de 2005.

Tabela 2 - N úmero de parasitóides $(\mathrm{N})$, riqueza de espé cies $(\mathrm{S})$ eíndices Shannon-Wiener $\left(\mathrm{H}^{\prime}\right)$, dediversidadede Simpson (1-D) e probabilidade (P) nos pomares de 'Montenegrina' e 'Murcott', comparados entre os anos julho de2003ajunho de2004(A no 1) ejulho de2004ajunho de 2005 (A no 2), Montenegro, RS.

\begin{tabular}{|c|c|c|c|c|c|c|}
\hline & \multicolumn{3}{|c|}{ ‘Montenegrina' } & \multicolumn{3}{|c|}{ 'Murcott' } \\
\hline & Ano 1 & Ano 2 & $P$ & Ano 1 & Ano 2 & $P$ \\
\hline $\mathrm{N}$ & 59 & 257 & - & 22 & 136 & - \\
\hline S & 2 & 5 & - & 1 & 5 & - \\
\hline $\mathrm{H}^{\prime}$ & 0,248 & 0,627 & 0,001 & 0 & 0,509 & 0,02 \\
\hline 1-D & 0,126 & 0,313 & 0,01 & 0 & 0,217 & 0,04 \\
\hline
\end{tabular}

Elasmus é um gênero cosmopolita, com espécies relacionadasaumaamplavariedadedehospedeiros, principalmente de Lepidoptera e Hymenoptera; podem, também, atuar como hiperparasitóides(ScHAUFF et al., 1998). Espécies deste gênero foram relatadas sobre P. citrella na Colômbia (CAstaño et al., 1996), Japão eTailândia (UJIYE etal., 1996), México (BAutısTAMARTINEZ et al., 1996), Itália (MINEO, 1999) e Brasil (SÁ et al., 1999; N A scimento etal., 2000; Montes et al .,2001).

A s espécies deC hrysocharis são endoparasitóides primários de larvas ou pupas de insetos minadores (Diptera, Lepidoptera, Coleoptera eH ymenoptera) e, de maneira geral, são solitários apresentando uma ampla gama de hospedeiros (H ANSSON, 1985a;b). Espécies deC hrysocharis associadas comP . citrellaforam relatadas no Japão (HEPPNER, 1993; UJIYE et al. 1996), Honduras (CAVE, 1996), Espanha (URBAneja et al., 1998), Ilhas Canárias, China, Chipreelsrael (SchaufF et al., 1998). Chrysocharis vonones encontrada no presente estudo ér eferida atacando principalmente larvas de moscas minadoras da família Agromyzidae (Acosta; Cave, 1994; Salvo; Valadares, 1997). No Brasil, já foi constatada parasitando P hyllocnistis sp. (Costa; Pereira, 2001). Entretanto, o registro do presente estudo constitui-se no primeiro sobre $P$. citrella

A . citricola éum endoparasitóide poliembriônico, podendoemergir, decadaminador parasitado, deum a oito indivíduos(A RGov R öSSLER, 1996). Suaintrodução foi considerada prioritária em vários países em função de sua liberação ter sido bem avaliada, especialmente na Austrália (N ELAE et al.,1995).

Em relação à diversidade de parasitóides nos 2 anosdeestudo, evidenciaram-sediferençassignificativas (Tabela 2), entretanto, entre as variedades num mesmo ano, não foram verificadas diferenças (A no 1: Shannon-Wiener - $P=0,18$, Simpson - $P=0,23$; A no 2: Shannon-Wiener $-P=0,27$, Simpson $-P=0,10$ ). Os dois períodos de estiagem registrados de julho de 2003ajunho de2004(A no 1), um nasaída do inverno e outro duranteo verão, que afetaram a fenologia da planta, o minador (hospedeiro) e as populações de parasitóides, podem explicar a diferença entre os anos. Conforme Gravena (1996), um inverno seco afeta a brotação deflorada doscitros (primeirofluxo), reduzindo o recurso disponível para o minador, cuja densi dade neste momento ainda é baixa. 
Tabela 3 - Coeficientes de correlação de Pearson dos números de parasitóides e câmaras pupais de Phyllocnistis citrella com rel ação aos fatores bióticos e abióticos, nas áreas de ‘Montenegrina' e ‘M urcott', M ontenegro, RS (julho de 2003a junho de 2005).

\begin{tabular}{llcccc}
\hline Fatores & \multicolumn{2}{c}{ 'Montenegrina' } & & \multicolumn{2}{c}{ 'Murcott' } \\
\cline { 2 - 3 } & Parasitóides & Câmaras pupais & Parasitóides & Câmaras pupais \\
\hline Temperatura média $\left({ }^{\circ} \mathrm{C}\right)$ & $0,3066^{*}$ & $0,4077^{* *}$ & & $0,2844^{*}$ & $0,4011^{* *}$ \\
Temperatura máxima $\left({ }^{\circ} \mathrm{C}\right)$ & $0,3410^{*}$ & $0,4346^{* *}$ & & $0,3025^{*}$ & $0,4188^{* *}$ \\
Temperatura mínima $\left({ }^{\circ} \mathrm{C}\right)$ & $0,3013^{*}$ & $0,3628^{* *}$ & & $0,2945^{*}$ & $0,3662^{* *}$ \\
Pluviosidade $(\mathrm{mm})$ & $-0,1359$ & $-0,0862$ & & $-0,0798$ & $-0,0317$ \\
Câmaras pupais & $0,8730^{* *}$ & - & $0,8907^{* *}$ & - \\
Brotos da quinzena anterior & 0,0756 & 0,0309 & & 0,0537 & 0,0117 \\
Brotos da quinzena anterior (a partir do & $0,3234^{*}$ & $0,3382^{*}$ & & $0,3724^{* *}$ & $0,4187^{* *}$
\end{tabular}

*Significante a $5 \%$.

**Significante a $1 \%$.

Comrelaçãoaosfatoresbióticos, nos2anos, observou-seuma dependência do número deparasitóides da quantidadedecâmaras pupais presentes nas áreas decitros (' $M$ ontenegrina' - ano $1: R^{2}=66,87 \%, P<$ 0,0001; ano 2: $\mathrm{R}^{2}=83,74 \%, \mathrm{P}<0,0001$; 'M urcott' - ano 1: $R^{2}=73,31 \%, P<0,0001$; ano $2: R^{2}=77,84 \%, P<$ 0,0001). Do mesmo modo, URBAneja et al. (2000), na Espanha, constataram que o parasitismo foi dependente da disponibilidade de hospedeiro e que os eulofídeos, emsuamaioriageneralistas, rapidamente aceitam P. citrella como novo hospedeiro, respondendo às variações na densidade populacional deste.

Considerando-seo período do experimento como um todo, os fatores abióticos registrados se correlacionarampositivamentedemaneirasignificativa com o número de parasitóides, à exceção da pluviosidade (Tabela 3). Utilizando-se a análise de regressão múltipladestes fatores evidenciou-sequea temperaturamáximafoi ofator demaior influênciano número de parasitóides registrados tanto em 'Montenegrina' $\left(R^{2}=11,63 \% ; P=0,0134\right)$ quanto em 'Murcott' ( $\left.R^{2}=8,90 \% ; P=0,031\right)$. Entretanto, os valores de $R^{2}$ foram baixos, o que pode indicar que 0 número de parasitóides responde a um modelo multifatorial, incluínd o fatores como umidaderelativa, presença deinimigos naturaise, principalmente, disponibilidade de hospedeiros.

NaÍndia, RAo; SHIVANKAR (2002) observaramqueo parasitismo em P. citrella por eulofídeosfoi negativamentecorrelacionado com a temperatura mínimaea velocidade do vento.

No presenteestudo constatou-se correl ação positiva entre o número de câmaras pupais e as médias das temperaturas médias, máximas e mínimas do período anterior à amostragem nas duas variedades. A temperatura máxima foi o fator que mais influenciou o número de câmaras pupais registradas, tanto em 'M ontenegrina' $\left(R^{2}=17,54 \%, P=0,002\right)$ como em
'Murcott' ( $\left.\mathrm{R}^{2}=18,89 \%, \mathrm{P}=0,0013\right)$, diferentementeda associação positiva entre a temperatura mínima ea população de $P$. citrella em limeira, registrada por PATEL et al. (1994), naÍndia, onde a maior população larval ocorreu quando a temperatura mínima era maior que $18^{\circ} \mathrm{C}$. Estes resultados possivel menteocorreram, pois na região da Índia onde o estudo foi desenvolvido, somentenumperíododoanoastempe raturasmédiassãoinferioresa $30^{\circ} \mathrm{C}$, o quecorresponde aos meses de inverno. No restante do ano, quando a temperatura médiaésuperior $a 30^{\circ} \mathrm{C}$, não háregistro do minador-dos-citros, o que sugereser esteum fator limitanteparaa espécie, conformeobservaramKATOLE et al. (1997) na Índia.

Em relação ao número de câmaras pupais do minador, não se verificou, tanto em 'M ontenegrina' quanto em 'Murcott', correlação significativa com a pluviosidade(Tabela3). NaÍndia, emlimeira'Kagzi', PATEL; PATEL (2001) não verificaram efeito significativo datemperaturamáximaemédiasobreonúmerode lagartas de P. citrella. A intensidade luminosa e a evaporação, segundo estes autores, foram os fatores que mais influenciaram negativamente a população do minador ea precipitação, a temperatura mínima, a umidade relativa e a pressão de vapor correlacionaram-se positivamente com a população deste. Neste mesmo país, Rao; SHivankar (2002), em tangerineira'Nagpur' elimeiraácida, demonstraram que a incidência do minador está correlacionada positivamente com a temperatura máxima, mínima, pluviosidade e velocidade do vento e de maneira negativa com a umidaderelativa, vapor de pressão e horas-luz.

Segundo PA tel et al . (1994), efeitos do número dehorasluz, temperatura mínima evapor depressão sobre P. citrella, quando testados individualmente, são mais pronunci ad osquequand o combinados. Assim, os val ores relativamente baixos de 
$\mathrm{R}^{2}$ obtidos através de regressão linear múltipla, no presente estudo, refletem a influência de fatores como a presença de predadores, umidade relativa eaqualidadedaplantahospedeira, sobreo número de câmaras pupais de P. citrella. De acordo com VARGAS et al. (2001), a qualidade da planta hospedeirainfluencia na acumulação deunidadestérmicas para o desenvolvimento de $P$. citrella, assim, plantas com maior quantidade de fotoassi milados em suas brotações são mais suscetíveis ao ataque do minador. Isto talvez explique a diferença no número de câmaras pupais entre os dois anos e entre as variedades, já queem ambos os anos registraram-se períodos de estiagem, os quais segundo Berlato (2005), foram os dois maiores dos últimos 50 anos no estado.

Além disso, as variedades 'Montenegrina' e 'Murcott' apresentam alternância de produção (Koller, 1994; Spósitoetal., 1998,R odrigues; Dorneles, 1999), fato quepodeter diminuído o recurso disponível para o minador entre os anos e, desta forma, influenciado suas populações.

Osvalores detemperaturaforam os mais importantes para o aparecimento e aumento da população de P. citrella no início de seu ciclo na área, entretanto apósseu estabel ecimento, a presença de brotos e os inimigos naturais parecem ser determinantes na flutuação do minador. Segundo BINGLIN; MINGDu (1996), na China, as variáveis meteorológicas são fatores-chave para as populações deP. citrella que ocorrem no final do outono, inverno e primavera.

De acordo com TiRADo (1995), na Espanha, em diferentes variedades de citros, o primeiro fluxo de brotação (primavera) pode representar mais de 60\% da nova área foliar anual eé, segundo o autor, pouco atacado por P. citrella. Estesresultadosforamcorroborados pelos de Generalitat Valenciana (1996) e URBANEjA et al. (2000) que não registraram ataque do minador no primeiro fluxo debrotação do citros (primavera), assim como no presente estudo, onde o primeiroemaiorfluxodebrotaçãodasplantasescapa da ação do minador.

Uma infestação variável deP. citrella nos diferentesfluxos debrotação em função defatores climáticos (temperaturas máximas, mínimasepluviosidade) foi verificada por RAo et al. (2002), de acordo com estes autores, no primei ro fluxo acorrelação foi positiva, no segundo não houve correlação eno terceiro foi negativa, evidenciando, conforme ocorrido no presente estudo, queas variáveismeteorológicasquecontribuemparaapresençado minador na brotação deprimavera (1o fluxo), acabam não contribuindo na última brotação (3o fluxo, verão-outono).

No inverno, no presenteestudo, não seconstatou a presença decâmaras pupais deP. citrella edeminas ativas em função da ausência de brotos. Este aspecto foi observado por DoumAndJI-MITICHE et al. (1999), na A rgélia, queconstataram uma diminuição no ataque deP. citrella no inverno eprimavera, devido às condições climáti cas desfavoráveis ea ausência de brotos; neste estudo apenas o parasitóide A. citricola, foi encontrado; as demais espécies de parasitóides, por serem generalistas, poderiam utilizar outros hospedeiros.

Com basenos resultados obtidos verificou-seque a ocorrência de parasitóides deP. citrella esteveassociada positivamente aos valores de temperatura eà presençadehospedeiro. Além disso, a diversidadede espécies de parasitóides não foi influenciada pela variedadedecitrosna qual o hospedeiro está presente; fatoresabióticoscomoatemperatura podemalterar tanto a diversidade como o número deindivíduos de um ano para o outro.

\section{AGRADECIMENTOS}

Ao Dr. Valmir A. Costa, do Instituto Biológico de Campinas(SP), pelaidentificação dos parasitóides; à Coordenação de A perfeiçoamento de Pessoal de Nível Superior (Capes) eao Conselho Nacional de Desenvolvimento Científico eTecnológico (CN Pq) pelas bolsas concedidas ao primeiro e segundo autores, respectivamente.

\section{REFERÊNCIAS}

Acosta, N.M.; CAVE, R.D.Inventario delos parasitoidesde Liriomyza spp. (Diptera: A gromyzidae) en la región sur deH onduras. R evista deBiologia Tropical, v.42,n.1/ 2, p.203-218, 1994.

AmARAL, A.M. Cancro cítrico: permanente preocupação da citriculturano Brasil enomundo. Brasília: EMBRAPA Recursos Genéticos e Agrobiologia, 2003. 5p. (EMBRAPA Recursos Genéticos e Agrobiologia. Comunicado Técnico, 86).

ARGOV, Y.; RöSSLER, Y. Introduction, releaseand recovery of several exotic natural enemies for biological control of the citrus leafminer Phyllocnistis citrella, in Israel. Phytoparasitica, v.24, n.1, p.33-38, 1996.

Bautista-M artinez, N .; Carrillo-Sanchez,J.L.;Bravo-Mojica, H.; Romero, J.; Pineda, S. Native parasitoids of the citrus leaf miner found at Cuitlahuac, Veracruz, México. In: Hoy, M.A. (Ed.). M anaging the citrus leafminer: Proceedingsof International Conference of Citrus Leafminer, Orlando, Florida, 1996. Gainesville: University of Florida, 1996. p.73.

Berlato, M.A. Prognósticos e recomendações para o período julho/agosto/setembro 2005. Disponível em: 丸ttp:/ / www.emater.tche.br/ site/ destaques/ ptbr/ forum tempoclima/ reuniaocopaaergs7-05-072005.pdf >. A cesso em: 7 dez. 2005. 
BINGLIN, T.; MINGDU, H. Managing the citrus leafminer in China. In: $\mathrm{H}$ or, M.A. (Ed.).M anaging thecitrusleafminer: Proceedings of International Conference of Citrus Leafminer, Orlando, Florida, 1996. Gainesville: University of Florida, 1996. p.49-51.

CALlegari-JACQUeS, S.M. Bioestatística: princípios e aplicações. Porto Alegre: ArtMed, 2003. v.1, 255p.

Castaño, O.; Garcia, R.F.; Trochez, A.; Rojas, L.; Peña, J.E.; Evans, G. Biological control of the citrus leafminer, Phyllocnistis citrella, in Colombia. In: Hor, M.A. (Ed.). $M$ anaging the citrus leafminer: Proceedings of International Conference of Citrus Leafminer, Orlando, Florida, 1996. Gainesville: University of Florida, 1996. p.76.

CAVE, R.D. Biological control of citrus leafminer in Honduras. In: Hor, M.A. (Ed.). M anaging the citrus leafminer: Proceedingsof International Conference of CitrusLeafminer, Orlando, Florida, 1996. Gainesville: University of Florida, 1996. p.78.

Chagas, M.C.M.; Parra, J.R.P.; N amekata, T.; Hartung, J.S.; Yamamoto, P.T. Phyllocnistis citrella Stainton (Lepidoptera:Gracillariidae) and itsrelationship with the citrus canker bacterium $X$ anthomonas axonopodis pv. citri in Brazil. Neotropical Entomology, v.30, n.1, p.55-59, 2001.

Costa, V.A.; Pereira, C.F. Ocorrência de Phyllocnistis sp. (Lep.: Gracillariidae) e seus parasitóides (Hym.: Chalcidoidea) em buva ( onyza bonariensis). In: SIMPÓSIO DE CONTROLE BIOLÓGICO, 7., 2001, Poços de Caldas. Resumos. Curitiba: PJ Eventos, 2001. p.322.

Diez, P.A.; Fidalgo, P. Cirrospilus neotropicus sp. n (Hymenoptera: Eulophidae): an indigenous biocontrol agent of the citrus leafminer,Phyllocnistis citrella (Lepidoptera: Gracillariidae). Entomological N ews, v.114, n.2, p.98-104, 2003.

Doumandi-Mitiche, B.; Chahbar, N.; Saharaoui, L. Survey of the population dynamics and the parasitic complex of Phyllocnistis citrella Stainton, 1856 (Lepidoptera: Gracillariidae) on two species of citrus in the region of Rouiba (Algiers). $M$ ededelingen Faculteit $L$ andbouwkundige en Toegepaste Biologische W etenschappen U niversiteit G ent, v.64, n.3, p.155-162, 1999.

GariJo, C.; García, E.J. Phyllocnistis citrella (Stainton, 1856) (Insecta: Lepidoptera: Gracillariidae: Phyllocnistidae) en los cultivos decítricos deA ndalucía (Sur España): Biología, ecología y control de la plaga. Boletin de Sanidad V egetal Plagas, v.20, n.4, p.815-826, 1994.

Generalitat Valenciana. El minador delas hojas delos cítricos (Phyllocnistis citrella St.). Valencia: Conselheria de A gricultura Y M edio Ambiente, 1996. 8p.

Graham, J.H.; Gottwald, T.R.; Browning, H.W.;A chor, D.S. Citrusleafminer exacerbated theoutbreak of A siatic citrus canker in South Florida. In: Hor, M.A. (Ed.). $M$ anaging the citrus leafminer: Proceedings of International Conference of Citrus Leafminer, Orlando, Florida, 1996. Gainesville: University of Florida, 1996. p.83.

Gravena, S. Lagartaminadoradoscitrosno Brasil.Laranja, v.17, n.1, p.286-288, 1996.
Hammer, Ø.; Harper, D.A.T.; Ryan, P.D. PAST: Paleontological Statistics Software Package for Education and Data A nalysis. PalaeontologiaElectronica, v.4, n.1, 2001. 9p.

Hansson, C. Taxonomy and biology of thePalearticspecies of Chrysocharis Förster, 1856 (Hymenoptera: Eulophidae). Entomologica Scandinavica Supplement, v.26, p.1-130, 1985a.

Hansson, C. Revised key to the Neartic species of Chrysocharis Förster (Hymenoptera: Eulophidae), including three new species. Journal $\mathrm{H}$ ymenoptera Research, v.4, p.80-98, 1985b.

HePPNER, J.B. Citrus leafminer, Phyllocnistis citrella, in Florida (Lepidoptera: Gracillariidae: Phyllocnistinae). Tropical Lepidoptera, v.4, n.1, p.49-64, 1993.

HINTZE, J. N CSS and PASS - Number cruncher statistical systems. Kaysville, Utah. Disponível em: łtttp:/ / www.ncss.com>. A cesso em: 2004.

Hoy, A.M.; NGUYEN, R. Classical biological control of the citrus leafminer Phyllocnistis citrella Stainton. Tropical Lepidoptera, v. 8, n.1, p.1-20, 1997.

IPAGRo - Instituto de Pesquisas Agronômicas. Atlas agroclimático do Estado do Rio Grande do Sul. Porto Alegre: Seção de Ecologia Agrícola, IPA GRO, 1989. 3V.

Jahnke, S.M.; Redaelli, L.R.; Diefenbach, L.M.G. Primeiro registro da ocorrência deCirrospilus floridensis Evans (Hymenoptera) como parasitóide de Phyllocnistis citrella Stainton (Lepidoptera) no Brasil.CiênciaRural, v.35, n.2, p.459-461, 2005a.

JahnKE, S.M.; RedaelLI, L.R.; DiefenbaCh, L.M.G. Complexo de parasitóides de Phyllocnistis citrella (Lepidoptera, Gracillariidae) em dois pomares de citros em Montenegro, RS, Brasil.Iheringia, v.95, n.4, p. 359-363, 2005b.

JahnKe, S.M.;RedaelLI, L.R.;DIefenbaCh, L.M.G.Parasitismo de Phyllocnistis citrella Stainton (Lepidoptera: Gracillariidae) em pomares de citros em Montenegro, RS. N eotropical Entomology, v. 35, n. 3, p. 357-363, 2006.

Katole, S.R.; Ughade, R.G.; Ingle, H.V.; Satpute, U.S. Effect of weather parameters on the incidence of citrus leafminer (Phyllocnisits citrella Stainton). PKV R esearch Journal, v.21, n.2, p. 252-253, 1997.

KolLeR, O.C.Citricultura: Iaranja, limão etangerina. Porto Alegre: Rigel, 1994. 446p.

KREBS, C.J. Ecological methodology. Menlo Park: Benjamin Cummings, 1998. 581p.

LaSalle, J.; Peña, J.E. A new species of Galeopsomyia (Hymenoptera: Eulophidae: Tetrastichinae): a fortuitous parasitoid of the citrus leafminer, Phyllocnistis citrella (Lepidoptera: Gracillariidae). Florida Entomologist, v.80, n.4, p.461-470, 1997.

Legaspi, J.C.; French, J.V.; Schauff, M.; Woolley, J.B. The citrus leafminer Phyllocnistis citrella (Lepidoptera: Gracillariidae) In South Texas: incidence and parasitism. Florida Entomologist, v.82, n.2, p.305-314, 1999.

MALUF, J.R.T. Nova classificação climática do Estado do Rio Grandedo Sul.RevistaBrasileira deA grometeorol ogia, v.8, n.1, p.141-150, 2000. 
MINEO, G. Records on indigenousantagonists of Phyllocnistis citrella (Lepidoptera: Gracillariidae) new for Italy. Bollettino di Zoologia A graria e Bachicoltura, v.31, n.1, p.97-105, 1999.

Montes, S.M.N.; Boliani, A.C.; Papa, G.; Cerávolo, A.; Rossi, A.C.; Namekata, T. Ocorrência de parasitóides da larvaminadoradoscitrosP hyllocnistis citrellaStainton, no Município dePresidentePrudente, SP.A rquivos do Instituto Biológico, São Paulo, v.68, n.2, p.63-66, 2001.

Moreno, C.E. M étodos para medir la biodiversidad. Zaragoza: M\&T - Manuales y Tesis SEA, 2001. 84p.

Nascimento, F.N.; Santos, W.; Pinto, J.M.; Cassino, P.C.R. Parasitismo em larvas deP hyllocnistis citrellaStainton (Lepidoptera: Gracillariidae) no Estado do Rio de Janeiro. A nais da Sociedade Entomol ógica do Brasil, v.29, n.2, p.377-379, 2000.

Neale,C.;Smith, D.;Beattie, G.A.C.;M iles, M. Importation, host specificity testing, rearing and realease of three parasitoids of Phyllocnistis citrella Stainton (Lepidoptera: Gracillariidae) in Eastern Australia. Journal of the Australian Entomological Society, v.34, p.343-348, 1995.

Paiva, P.B.; Gravena, S.; Amorim, L.C.S. Introdução do parasitóide A geniaspis citricola Logvinovskaya para controlebiológico da minadora dasfolhas doscitros Phyllocnistis citrella Stainton no Brasil. Laranja, v.29, n.1, p.149-154, 2000.

Patel, N.C.; V aland, V.M.;Shekh,A.M.;Patel, J.R. Effect of weather factors on activity of citrus leaf-miner (Phyllocnistis citrella) infesting lime(Citrusaurantifolia). Indian Journal of A gricultural Science, v.64, n.2, p.132134, 1994.

Patel, G.P.;PAtel, J.R. Population dynamics ofPhyllocnistis citrella on citrus in middle Gujarat. Indian Journal of Entomology, v.63, p.41-48, 2001.

Pomerinke, M.A.;Stansly, P.A. Establishment ofA geniaspis citricola (Hym., Encyrtidae) for biological control of Phyllocnistis citrela (Lep., Gracillaridae) in Florida. Florida Entomologist, v.81, n.3, p.361-372, 1998.

Prates, H.S.; Nakano, O.; Gravena, S.A. A "minadora das fol has decitros" Phyllocnistis citrella Stainton 1856. Campinas: CATI, 1996. p.2-8. (ComunicadoTécnico CATI, 129).

RaO, C.N.; SHIVAnKar V.J. Incidence of citrus leaf miner (Phyllocnistis citrella) and itsnatural enemies in central India. Indian Journal of A gricultural Science, v.72,p.625627, 2002.

RaO, K.R.; PAthak, K.A.; SHyLEShA, A.N. Spatio-temporal changes in the infestation of citrus leafminer, Phyllocnistis citrella Stainton in Meghalaya. Entomon, v.27, n.2, p.169-178, 2002.
Rodrigues, L.R.; Dornelles, A.L.C. Origemecaracterização horticultural da tangerina 'M ontenegrina'. Laranja, v.20, n.1, p.153-166, 1999.

Sá, L.A.N.; Costa, V.A.; Tambasco, F.J.; Qiveira, W.P.; ALmEIDA, G.R. Parasitóides da larva minadora da fol ha dos citrus, P hyllocnistis citrella Stainton, estudos no laboratório de quarentena "Costa Lima" em Jaguariúna, SP. Jaguariúna: EMBRAPA Meio Ambiente, 1999. p.1718. (EMBRA PA M eio A mbiente. Comunicado Técni$\mathrm{CO}, 2)$.

Salvo, A.; Valladares, G.R. A $n$ analysis of leaf-miner and plant host ranges of threeC hrysochar is species(Hym.: Eulophidae) from A rgentina. Entomophaga, v.42, n.3, p.387-396, 1997.

Schauff, M.E.; Lasalle, .; W IJESEKARA, G.A. Thegenerao the Chalcid parasitoids (Hymenoptera: Chalcidoidea) of citrus leafminer Phyllocnistis citrella Stainton (Lepidoptera: Gracillariidae). J ournal of N atural H istory, v.32, p.1001-1056, 1998.

Spósito, M.B.; CAstro, P.R.; A gustı, M. Alternância de produção em citros. Laranja, v.19, n.2, p.285-292, 1998.

TIRADO, L.G. Dañoscausadospor losataquesdePhyllocnistis citrella Stainton (Lepidoptera: Gracillariidae), y su repercusión sobrelaproducion deárbolesadultosde cítricos. Boletin deSanidad V egetal Plagas, n.23, p.73-91, 1995.

UjIYe, T.; KamiJo, K.; M orakote, R. Species composition of parasitoid and parasitism of the citrus leafminer (CLM), Phyllocnistis citrella Stainton (Lepidoptera, Gracillariidae) in central and northern Thailand, with key of parasitoids of CLM collected fromJapan, Taiwan and Thailand.Bulletin of theF ruit TreeR esearch Station, v.29, p.79-106, 1996.

Urbaneja, A.; Jacas, J.; Verdú, M.J.; Garrido, A. Dinámica e impacto delos parasitoidesautóctonos dePhyllocnistis citrella Stainton, en comunidad valenciana. Investigación A grária: producción y protección vegetales, v.13, n.3, p.409-423, 1998.

Urbaneja, A.; LÁcer, E.; TomÁs, Ó.; Garrido, A.; JacAs, J. Indigenous natural enemies associated with Phyllocnisits citrella (Lepidoptera: Gracillariidae) in Eastern Spain. Biological Control, v.18, p.199-207, 2000.

Vargas, H.A.; Bobadilla, D.E.; Vargas, H.E. Thermal requirements for ontogenic development of Phyllocnistis citrella Stainton (Lepidoptera: Gracillariidae). Idesia, v.19, p.35-37, 2001.

Recebido em 2/ 8/ 06

Aceito em 12/ 4/ 07 